\title{
Evaluation of $\mathrm{CT}$ vascularization patterns for survival prognosis in patients with hepatocellular carcinoma treated by conventional TACE
}

\author{
Davut B. Hasdemir \\ Lukas Aguirre Dávila \\ Nora Schweitzer \\ Bernhard C. Meyer \\ Armin Koch \\ Arndt Vogel \\ Frank Wacker \\ Thomas Rodt
}

From the Institutes of Diagnostic and Interventional Radiology (D.B.H., B.C.M., F.W., T.R. $\$ rodt.thomas@ mh-hannover.de and Biometry (L.A.D., A.K.), the Department of Gastroenterology, Hepatology and Endocrinology (N.S., A.V.), The Hannover Medical School, Hannover, Germany.

Received 17 February 2016; revision requested 3 April 2016; last revision received 29 June 2016; accepted 28 August 2016.

Published online 3 March 2017. DOI 10.5152/dir.2016.16006

\begin{abstract}
PURPOSE
Transarterial chemoembolization (TACE) is an established treatment for intermediate stage hepatocellular carcinoma (HCC). The aim of this retrospective study was to evaluate the power of lesion vascularization criteria based on computed tomography for prognosis of overall survival before initiation of treatment.
\end{abstract}

\section{METHODS}

A total of 59 patients with intermediate stage HCC treated with TACE as first-line treatment were retrospectively evaluated. TACE procedures were performed using doxorubicin, cisplatin, and lipiodol. Response evaluation criteria in solid tumors version 1.1 (RECIST 1.1) were used to determine the initial tumor response. Four vascularization patterns (VP) of the largest target lesion (homogeneous vascularization [VP1], homogeneous vascularization with additional arterial hypervascularization [VP2], heterogeneous vascularization with [VP3] and without zones of hypervascularization [VP4]) were assessed prior to the first TACE and correlated to survival.

\section{RESULTS}

Kaplan-Meier analysis yielded a median overall survival of 608 days (standard error [SE], 120.5 days). Survival analysis showed significant differences depending on the vascularization patterns $(P=0.012$; hazard ratio, 0.327): patients with homogeneously vascularized lesions (VP1,VP2) had a median overall survival of 1091 days (SE, 235.5 days). Patients with heterogeneous vascularization of the lesion (VP3 and VP4) showed a median overall survival of 508 days (SE, 113.9 days).

\section{CONCLUSION}

The vascularization pattern of the largest HCC lesion is helpful for survival prognosis under TACE treatment and therefore has the potential to be used as an additional parameter for treatment stratification.

$\mathrm{T}$ ransarterial chemoembolization (TACE) is an established therapy for intermediate stage hepatocellular carcinoma $(H C C)$ mostly used in a palliative setting $(1,2)$. With publication of the Barcelona criteria in 2001 (Barcelona clinic liver cancer, BCLC), therapy guidelines for individual tumor stages were established (3-5).

Even though TACE is widely accepted as an efficient treatment in clinical routine, scientific evidence for overall survival benefit has been variable. A very recent review published in 2015 by Sieghart et al. (6) states that the prevalent heterogeneity of treatment modalities is one of the major limitations for a clear statement considering survival benefit. TACE treatment response assessment based on imaging is frequently used as a surrogate. However, the prognostic value of this imaging based treatment success assessment is ambiguous. Modified response evaluation criteria in solid tumors (mRECIST) and the European Association for the Study of the Liver (EASL) criteria enable a survival prognosis as early as after second or third TACE (7). However, the use of these response assessments based on imaging has downsides since in cases of inhomogeneous lipiodol distribution with pinpoint scattering of lipiodol, these criteria are difficult to apply correctly in clinical practice (Fig. 1) (8). Moreover, an earlier assessment of prognosis of TACE outcome would be more relevant, as one could opt for alternative treatment options that are more effective or less quality of life threatening. Kawamura et al. $(9,10)$ proposed a four-fold vascularization pattern differentiation based on imaging depending on enhancement in the arterial and portovenous phases and correlated these with histologic findings after resection in early stage HCC and prediction of HCC recurrence. 
The purpose of this study was to investigate the power of a four-fold categorization of HCC vascularization patterns on baseline computed tomography (CT) to predict overall survival of patients treated by conventional TACE before treatment is started.

\section{Methods}

\section{Patients}

In this retrospective single center cohort study, patients who had their first conventional TACE between 2006 and 2008 were monitored throughout further clinical course until death or termination of the follow-up. Patients with prior systemic therapy or percutaneous ethanol injection (PEI) were excluded from the study, since persistent effects of the systemic therapy on healthy liver tissue and vessels could not be ruled out and PEI was not recommended according to BCLC guidelines since radiofrequency ablation (RFA) showed better tumor control with fewer interventions. While the $\mathrm{BCLC}$ criteria still viewed PEI as an option in 2003, even though inferior to RFA, it completely disappeared from the revised guidelines in 2011 for the treatment of intermediate stage $\operatorname{HCC}(4,8,11)$. For this reason, patients in our institution were not treated with $\mathrm{PEI}$ on a regular basis, and patients with prior PEI treatment were excluded for potential bias. Surgical resection or RFA and microwave ablation treatment before TACE were not considered as exclusion criteria. Seven patients receiving a liver transplant in the further course of TACE interventions were censored for survival analysis. All in-

\section{Main points}

TACE has been shown to increase overall survival in the treatment of intermediate stage HCC. As therapeutic response to TACE is variable, retrospective imaging-based response assessment is currently used to decide on individual further treatment.

In our retrospective study, biphasic CT-based vascularization parameters of the largest HCC target lesion prior to conventional TACE treatment were correlated to overall survival. A multivariate regression analysis of several potential prognostic parameters determined vascularization pattern and Child-score to be significant prognostic factors for overall survival.

The vascularization pattern of the largest HCC lesion before TACE treatment has potential to be used as an additional parameter for individual treatment stratification. cluded patients had follow-up imaging performed according to our institutional protocol. Follow-up was available until July 2012. The institutional review board approved this study and informed consent was collected from every patient.

A total of 59 patients (49 male and $10 \mathrm{fe-}$ male patients; mean age, 66.7 years) could be included in the study based on the inclusion criteria. Patients received a total of 135 TACE procedures within the complete follow-up time. Of the patients 49 (83.1\%) were categorized as Child-Pugh class A, 8 (13.6\%) as class B and $2(3.4 \%)$ as class C. HCC was diagnosed based on histology or the noninvasive EASL guidelines (4). However, histologic specimens were available for only $20 \%$ of the patient cohort $(n=12)$. Therefore no further analysis regarding histology was performed in this study.
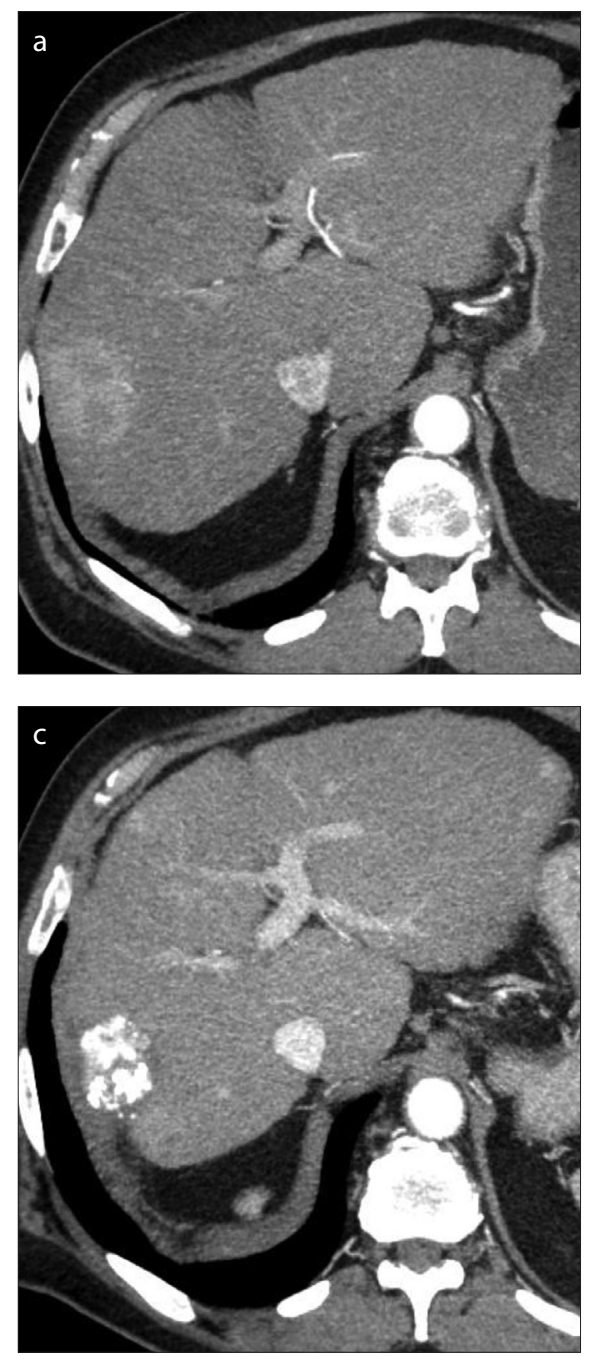

Figure 1. a-d. Hypervascularized hepatocellular carcinoma target lesion prior to initial transarterial chemoembolization (TACE) (a). After first TACE, slight accumulation of lipiodol is present (b). After second (c) and third (d) TACE procedures, a progressive lipiodol accumulation with pinpoint scattering pattern in combination with tumor shrinkage was observed.
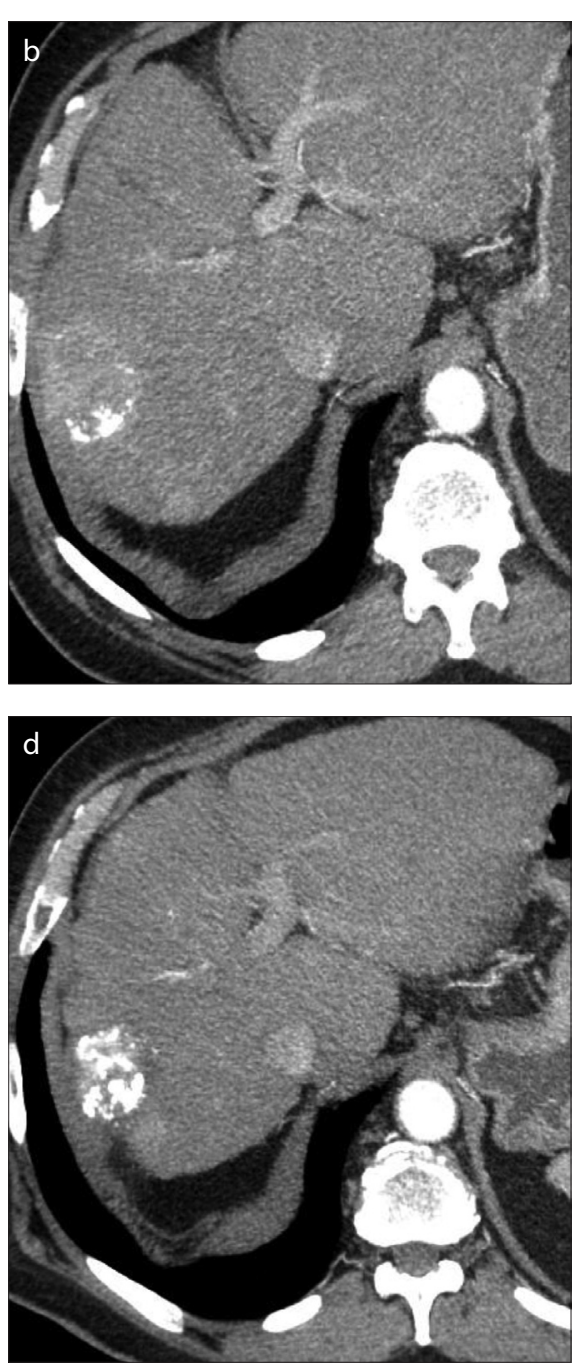

\section{TACE treatment}

All patients received a standardized conventional TACE protocol consisting of cisplatin (Cisplatin Teva, TEVA) and doxorubicin (DOXO-cell, Cell pharm) as chemotherapy and lipiodol as the only embolizing agent (Lipiodol Ultra Fluide; Laboratoire Guerbet). Cisplatin and doxorubicin doses were calculated based on body surface, cardiac and renal function, with a doxorubicin and cisplatin standard dose of $50 \mathrm{mg} / \mathrm{m}^{2}$ each. Echocardiography was performed to assess cardiac function and glomerular filtration rate was calculated to assess renal function. Depending on the scale of cardiac or renal function impairment doxorubicin/cisplatin dosages were reduced or the substances not administered. Lipiodol standard dose was $10 \mathrm{~mL}$ except in cases of extensive arterial hypervascularization of the target le- 


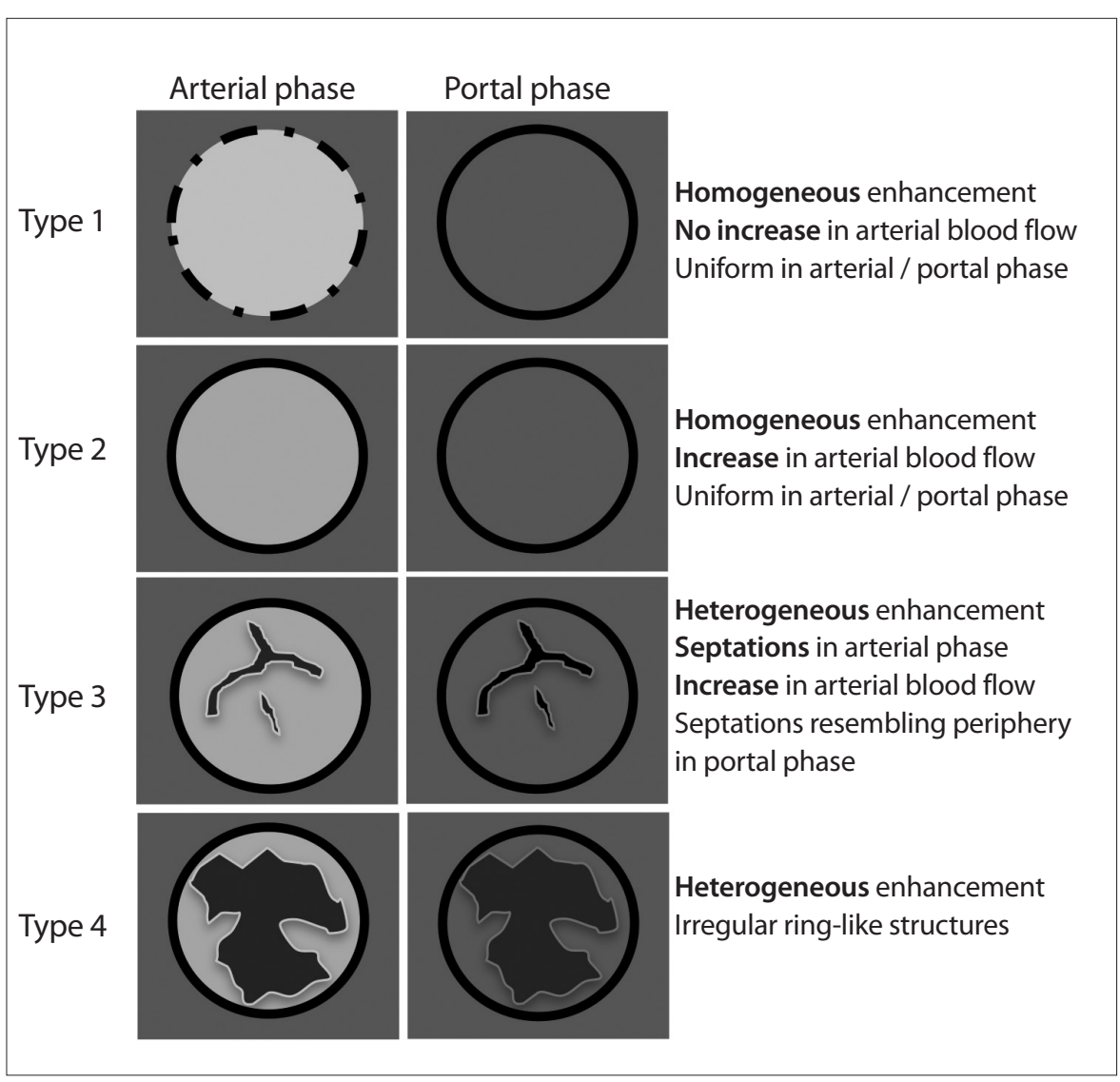

Figure 2. Vascularization patterns based on categorization by Kawamura et al. (9).

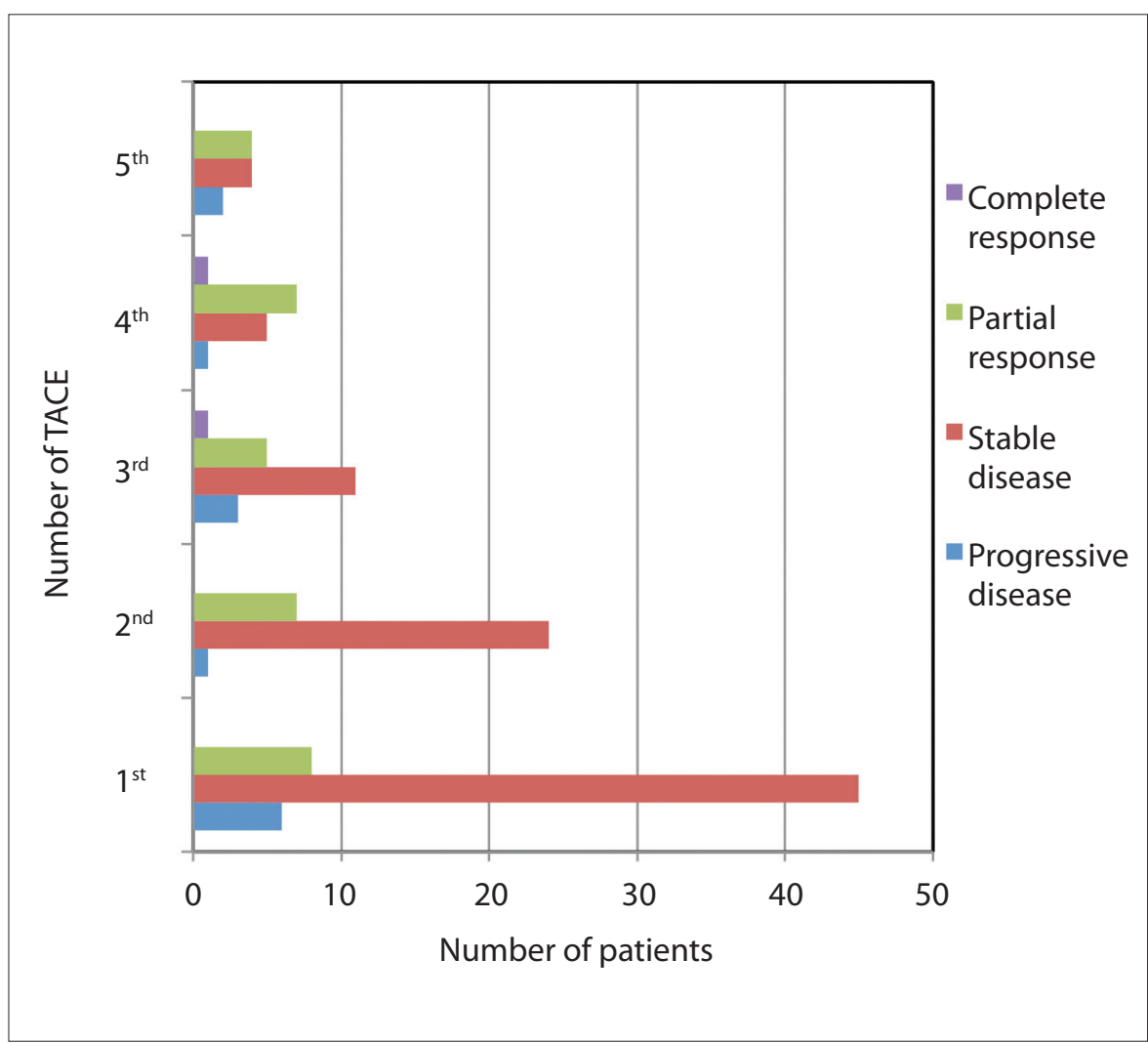

Figure 3. RECIST1.1 tumor response according to the individual TACE cycles. The horizontal axis shows the number of patients receiving chemoembolization. sion. In those cases additional lipiodol up to a maximum of $10 \mathrm{~mL}$ was administered. Median treatment interval between TACE procedures was 80 days (range, 48-730 days).

\section{Imaging}

Biphasic CT was performed using $100 \mathrm{~mL}$ of Imeron (Imeron350, Bracco Imaging) with slice thickness $\leq 5 \mathrm{~mm}$ at baseline within one week before TACE at the most. For response assessment and potential second TACE, a CT scan with exactly the same protocol was performed 2-3 months after the first intervention (median, 71 days; range, 40-700 days). Kawamura et al. (9) evaluated the vascularization of the largest target lesion based on the baseline imaging. The proposed categorization of vascularization patterns is summarized in Fig. 2. A lesion with a vascularization pattern 1 (VP1) shows homogeneous vascularization in arterial and portovenous phase without increased arterial blood flow, whereas a VP2 lesion also shows a homogeneous vascularization pattern but with increased arterial attenuation. A VP3 lesion shows increased arterial attenuation with septal structures in this phase. A VP4 shows heterogeneous vascularization with an irregular structure. In the arterial phase, tumor tissue in the periphery shows a ring-like vascularization, whereas the center of the lesion remains hypodense. In portal venous phase, the center still remains hypodense in contrast to the ringlike structure in the periphery of the lesion (Fig. 2). Response to TACE was evaluated according to RECIST1.1 criteria (Fig. 3).

\section{Statistical analysis}

For statistical analysis SPSS v. 21 (SPSS, IBM Inc.) was used. Duration of survival was expressed as median and standard error (SE). Kaplan Meier analysis was performed to descriptively assess overall survival. A Cox regression model was used to investigate the impact of sex, vascularization pattern, Child-Pugh score, age, and RECIST response assessment on overall survival. Parameters that showed association to overall survival in the univariate analysis with a significance level $P<0.15$ were included in a multivariate Cox regression model and evaluated using backward elimination (Wald test) with a significance level of $P<0.05$.

\section{Results}

Initial TACE treatment could be performed, the successfully in all 59 patients. On average, the patients received 2.9TACE treat- 


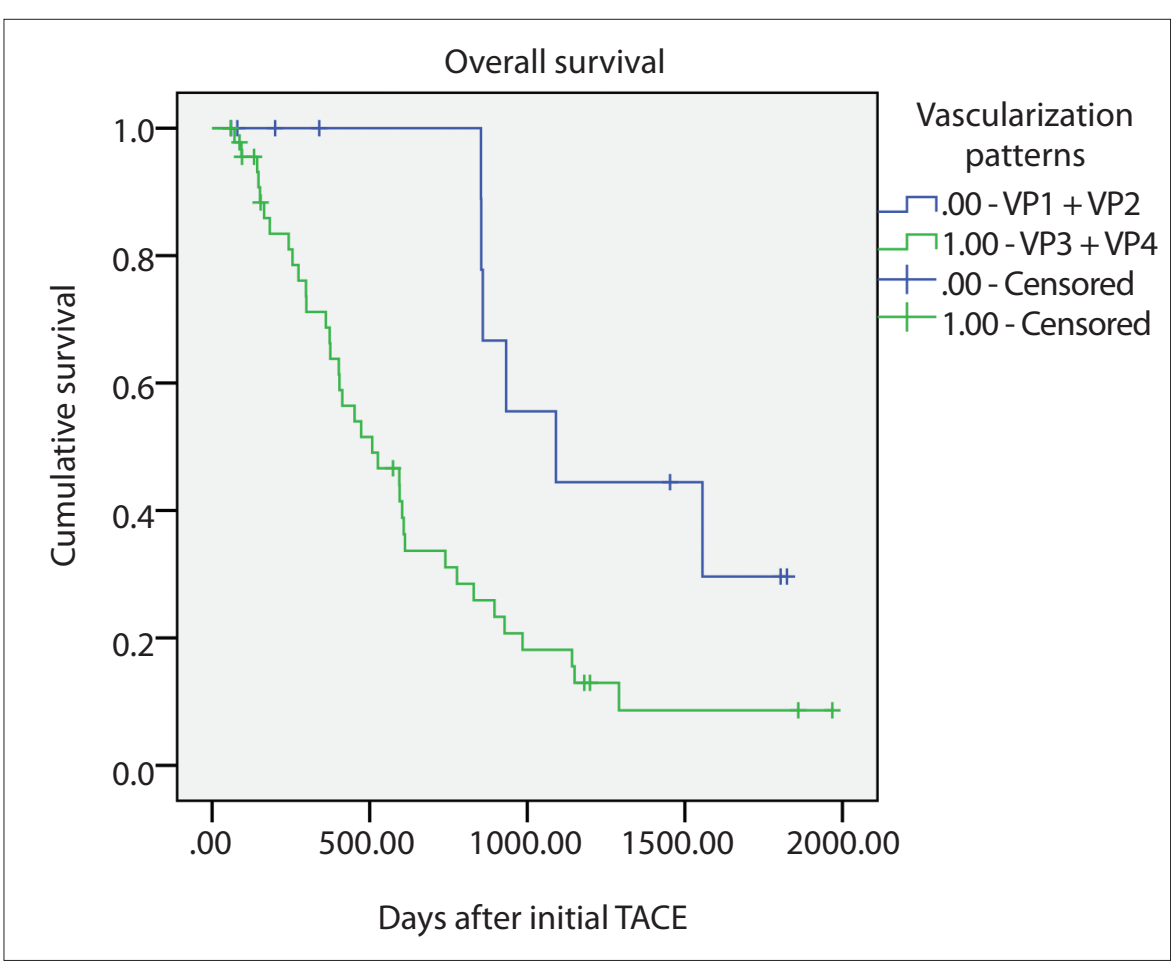

Figure 4. Overall survival according to vascularization pattern (VP). Patients with homogeneously vascularized (VP1+VP 2) target lesions (blue line) show a significantly higher survival rate than patients with heterogeneously vascularized (VP3+VP 4) target lesions (green line).

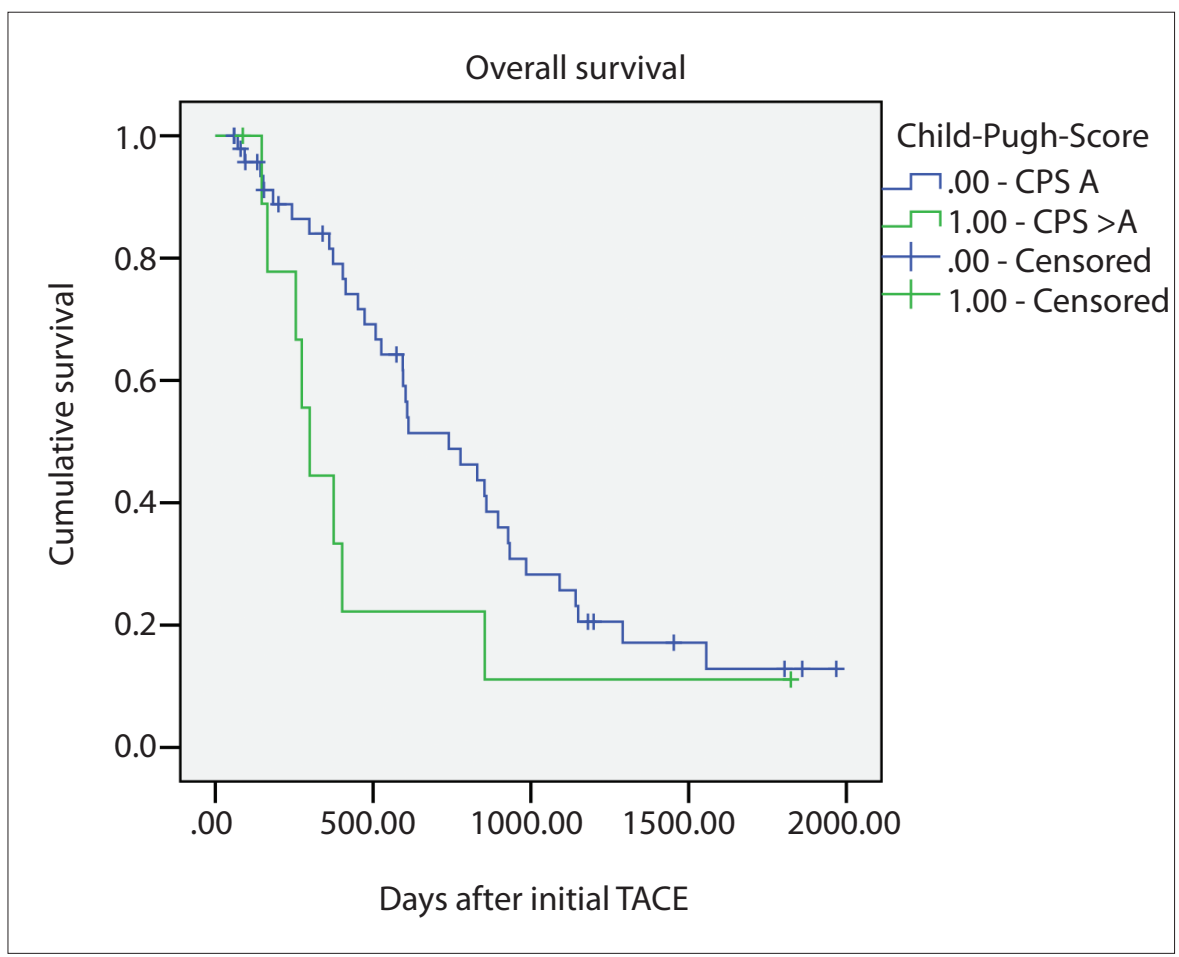

Figure 5. Overall survival according to Child-Pugh score. Patients with Child-Pugh class A (blue line) show a significantly higher survival rate than patients with Child-Pugh class B or C (green line).

ments with a maximum of 10 procedures. The median treatment interval was 80 days (range, 48-730 days). Minor adverse events according to the Society of Interventional Radiology guidelines for the reporting and archiving of interventional radiologic procedures were seen in 10 cases; these adverse events did not result in prolonged hospital stay or an increase in therapeutic measures (12).
Kaplan-Meier survival analysis yielded a median overall survival of 608 days (SE, 120.5 days). One-year survival rate was $68.8 \%$ and two-year survival rate was 40.6\%.

Patients presenting with a VP1 or VP2 target lesion had a median overall survival of 1091 days (SE, 235.5 days) following the first chemoembolization (Fig. 4). The remaining group of 46 patients showing a VP3 or VP4 had a median survival of 508 days (SE, 113.9 days). There was a significant difference in the survival rates of the groups with homogeneous enhancement of the target lesion (VP1, VP2) compared with the groups with heterogeneous enhancement of the target lesion (VP3, VP4) $(P=0.012$; hazard ratio [HR], 0.327). RECIST 1.1 baseline measurement also showed a significant difference for survival depending on the largest diameter of the largest target lesion $(P=0.039$; $H R, 0.520)$. Median RECIST 1.1 baseline measurement of the target lesion was $6.67 \mathrm{~cm}$. Patients with a lesion diameter less than the median diameter showed a median overall survival of 859 days (SE, 90.2 days), while patients with a diameter above median presented with a median overall survival of 473 days (SE, 82 days). Child-Pugh score did show a significant impact on survival. Patients with Child-Pugh class A $(n=49)$ had a median overall survival of 740 days (SE, 139.2 days), whereas the remaining 10 patients with Child-Pugh class B and C presented with a median overall survival of 299 days (SE, 37.3 days) (Fig. 5). Cox regression analysis yielded a HR of $0.508(P=0.089)$. Sex had a significant impact on overall survival ( $P=0.023$; HR, 0.367). Female patients had a lower median overall survival of 298 days (SE, 178.9 days) than that of male patients (777 days; $\mathrm{SE}=165.1$ days). Age did not have a significant impact on overall survival $(P=0.120 ; \mathrm{HR}, 1.625)$.

A Cox regression analysis using stepwise backward elimination including sex, vascularization pattern, Child-Pugh score, age and RECIST 1.1 as independent variables determined vascularization pattern $(P=$ 0.002; HR, 0.224) and Child-Pugh score $(P$ $=0.005 ; \mathrm{HR}, 0.278$ ) to be significant prognostic factors for overall survival, while age, sex and RECIST 1.1 baseline value were not significant.

\section{Discussion}

This study shows that the CT vascularization pattern of the largest HCC target lesion before the first conventional TACE 
treatment and Child-Pugh score show significant correlation to overall survival.

Our protocol proved to be within the range of current publications for conventional TACE concerning treatment intervals, the amount of procedures, and outcome. Patients in our cohort received a mean of $2.9 \pm 2.3$ interventions and the median interval was 80 days (range, 48730 days). Furthermore, tumor response was comparable to findings in the literature (13). Tumor control, defined as complete and partial response combined with stable disease is often used for compact description of response to treatment (14). Tumor control was achieved in $88.3 \%$ of all patients after first TACE. More details on tumor response are shown in Fig. 3. One-year survival was $68.8 \%$ and two-year survival was $40.6 \%$. Literature shows comparable overall survival rates with a median one-year survival rate of $62.5 \%$ using conventional TACE $(3,15,16)$.

For analysis and differentiation of factors facilitating prediction of overall survival before initial treatment, a Cox regression analysis was performed as reported. We included parameters, which we considered relevant to affect survival prognosis for patients undergoing repetitive conventional TACE. The first factors to be eliminated were age and RECIST baseline measurement, used as surrogate for pretreatment tumor burden. There are studies supporting this result whereas others show a significant impact of tumor size and lesion number on overall survival (17). A reason for the different findings in the literature could be a missing standard for evaluation of tumor burden, which is often evaluated based on the BCLC score and Okuda stage. The Okuda stage discriminates between more or less than 50\% liver involvement of the tumor. The BCLC score differentiates between single or multiple lesions and further differentiation depends on the diameter. Nevertheless many studies only distinguish between solitary or multinodular tumors and the mean size investigated in their own patient cohort (18). The next factor to be eliminated in our analysis was gender, leaving vascularization pattern and Child-Pugh score as the remaining significant factors for prognosis of overall survival in our TACE cohort. Various studies already suggested that Child-Pugh score is an important factor for prognosis of overall survival in TACE treatment $(4,19)$.

The analysis of CT vascularization patterns as reported by Kawamura et al. (9) showed a significantly higher overall survival for lesions with homogeneous vascularization pattern (VP1, VP2) compared with lesions with a heterogeneous vascularization pattern (VP3, VP4) (4). Kawamura et al. $(9,10)$ could not show a significant connection between the proposed vascularization patterns and overall survival in his initial studies. In contrast to our study with a palliative setting, the study by Kawamura et al. (9) included only patients with a curative resection after TACE. Therefore, most of the tumors were less advanced in stage compared with our cohort. The majority of the evaluated lesions (50\%) showed a VP4 accounting for extensive necrosis prior to conventional TACE in this study. The cohort in the study by Kawamura et al. (9) consisted mostly of VP1-VP3 and only $14 \%$ of the lesions were VP4. The poor differentiation of VP4 in histopathologic analysis explains the low survival of our patients with VP4 lesions with a median of 428 days (range, 93-1968 days) compared with patients with VP1 lesions of 1504.5 days (range, 853-1824 days) (9).

Besides vascularization patterns, there are other imaging criteria accounting for vascularization, the most commonly used being the mRECIST and EASL response assessment criteria. Both also rely on the difference between vascularized and non-vascularized regions of the tumor lesion (20, 21). Several studies showed that mRECIST can allow survival prognosis after the third or even the second TACE $(1,7,22)$. However, in our study the dichotomous differentiation of vascularization patterns facilitates survival prognosis even prior to the initial intervention. Thus, it has great potential as a parameter for treatment stratification early in the clinical course. A critical issue regarding conventional TACE and mRECIST or EASL response evaluation is the potential inhomogeneity of lipiodol distribution causing problems for a valid measurement $(8,17)$. In cases of pinpoint scattering of lipiodol one might have to move back to RECIST 1.1 criteria instead (23). mRECIST and EASL only assess the amount of the vascularized lesion part. However, other studies emphasize that not only size but also the type of vascularization and lesion composition is important (24).

This study has some limitations. Even though conventional TACE with lipiodol has previously been the standard of care, a recent analysis of interventional radiologists in Germany showed that $43.5 \%$ of all interventions were performed using drug eluting beads (DEB TACE), closely followed by conventional TACE in $42 \%$ of the procedures (25). Therefore this analysis should also be performed in patients treated with DEB TACE or bland embolization with microspheres alone. A recent study published by Brown et al. (26) showed that doxorubicin as a chemotherapeutic agent does not show a significant benefit for the patient in terms of response; similarly, earlier publications could not prove a benefit of a chemotherapeutic agent vs. bland embolization for a patients' overall survival $(3,27)$. Since the aspect of embolization draws more and more attention, future studies should compare the quantitative aspect of HCC vascularization with the qualitative judgment of the vascularization pattern analysis based on the categorization by Kawamura et al. (9). A further limitation is the qualitative visual analysis of lesions instead of a quantitative three-dimensional (3D) computed analysis of vascularization characteristics. Even though promising results have been achieved using 3D computed analysis, for everyday use it currently does not qualify as the standard procedure $(28,29)$. Additionally, a study published by Donati et al. (29) could prove that inter-test agreement between visual estimation and two-dimensional measurement of enhancing tissue is interchangeable, showing that estimation based assessment like the categorization by Kawamura et al. (9) is not a disadvantage in general, but a pragmatic approach towards clinical practice. Another limitation of this study is the rather small number of study subjects. The results of this analysis warrant further investigation in larger patient collectives.

In conclusion, CT vascularization pattern and Child-Pugh score are relevant prognostic parameters for overall survival since patients with heterogeneously vascularized target lesions showed a low overall survival rate when treated with conventional TACE in this study. Therefore, these parameters have a potential for treatment stratification at an earlier point in the clinical course than established vascularization assessment criteria such as mRECIST or EASL based on follow-up imaging.

\section{Conflict of interest disclosure}

The authors declared no conflicts of interest.

\section{References}

1. Cescon $M$, Cucchetti A, Ravaioli M, Pinna AD. Hepatocellular carcinoma locoregional therapies for patients in the waiting list. Impact on transplantability and recurrence rate. J Hepatol 2013; 58:609-618. [CrossRef] 
2. Park JW, Amarapurkar D, Chao Y, et al. Consensus recommendations and review by an International Expert Panel on Interventions in Hepatocellular Carcinoma (EPOIHCC). Liver Int 2013; 33:327-337. [CrossRef]

3. Marelli L, Stigliano R, Triantos C, et al. Transarterial therapy for hepatocellular carcinoma: which technique is more effective? A systematic review of cohort and randomized studies. Cardiovasc Intervent Radiol 2007; 30:6-25. [CrossRef]

4. Llovet JM, Fuster J, Bruix J, Barcelona-Clínic Liver Cancer Group. The Barcelona approach: diagnosis, staging, and treatment of hepatocellular carcinoma. Liver Transpl 2004; 10:115-20. [CrossRef]

5. Raoul JL, Sangro B, Forner A, et al. Evolving strategies for the management of intermediate-stage hepatocellular carcinoma: Available evidence and expert opinion on the use of transarterial chemoembolization. Cancer Treat Rev 2011; 37:212-220. [CrossRef]

6. Sieghart W, Hucke F, Peck-Radosavljevic M. Transarterial chemoembolization: modalities, indication, and patient selection. J Hepatol 2015; 62:1187-1195. [CrossRef]

7. Gillmore R, Stuart S, Kirkwood A, et al. EASL and mRECIST responses are independent prognostic factors for survival in hepatocellular cancer patients treated with transarterial embolization. J Hepatol 2011; 55:1309-1316. [CrossRef]

8. Bruix J, Sherman M. Management of hepatocellular carcinoma: An update. Hepatology 2011; 53:1020-1022. [CrossRef]

9. Kawamura Y, Ikeda K, Hirakawa M, et al. New classification of dynamic computed tomography images predictive of malignant characteristics of hepatocellular carcinoma. Hepatol Res 2010; 40:1006-1014. [CrossRef]

10. Kawamura Y, Ikeda K, Seko Y, et al. Heterogeneous type 4 enhancement of hepatocellular carcinoma on dynamic CT is associated with tumor recurrence after radiofrequency ablation. AJR Am J Roentgenol 2011; 197:665-673. [CrossRef]

11. Forner A, Llovet JM, Bruix J. Chemoembolization for intermediate HCC: is there proof of survival benefit? J Hepatol 2012; 56:984-986. [CrossRef]

12. Omary RA, Bettmann MA, Cardella JF, et al. Quality improvement guidelines for the reporting and archiving of interventional radiology procedures. J Vasc Interv Radiol 2002; 13:879881. [CrossRef]
13. Kim CJ, Kim HJ, Park JH, Park DI, et al. Radiologic response to transcatheter hepatic arterial chemoembolization and clinical outcomes in patients with hepatocellular carcinoma. Liver Int 2014; 34:305-312. [CrossRef]

14. Vogl TJ, Naguib NNN, Nour-Eldin N-EA, et al. Review on transarterial chemoembolization in hepatocellular carcinoma: Palliative, combined, neoadjuvant, bridging, and symptomatic indications. Eur J Radiol 2009; 72:505-516. [CrossRef]

15. Kothary N, Weintraub JL, Susman J, Rundback JH. Transarterial chemoembolization for primary hepatocellular carcinoma in patients at high risk. J Vasc Interv Radiol 2007; 18:1517-1526. [CrossRef]

16. Burrel M, Reig M, Forner A, et al. Survival of patients with hepatocellular carcinoma treated by transarterial chemoembolisation (TACE) using drug eluting beads. Implications for clinical practice and trial design. J Hepatol 2012; 56:1330-1335. [CrossRef]

17. Wu KT, Wang CC, Lu LG, et al. Hepatocellular carcinoma: clinical study of long-term survival and choice of treatment modalities. World J Gastroenterol 2013; 19:3649-3657. [CrossRef]

18. Lin M, Pellerin O, Bhagat N, et al. Quantitative and volumetric European Association for the Study of the Liver and Response Evaluation Criteria in Solid Tumors measurements: feasibility of a semiautomated software method to assess tumor response after transcatheter arterial chemoembolization. J Vasc Interv Radiol 2012; 23:1629-1637. [CrossRef]

19. Jin B, Wang D, Lewandowski RJ, et al. Chemoembolization endpoints: effect on survival among patients with hepatocellular carcinoma. AJR Am J Roentgenol 2011; 196:919-928. [CrossRef]

20. Takuma $Y$, Takabatake $H$, Morimoto $Y$, et al. Comparison of combined transcatheter arterial chemoembolization and radiofrequency ablation with surgical resection by using propensity score matching in patients with hepatocellular carcinoma within Milan criteria. Radiology 2013; 269:927-937. [CrossRef]

21. Abdel-Rahman O, Elsayed ZA. Combination trans arterial chemoembolization (TACE) plus sorafenib for the management of unresectable hepatocellular carcinoma: a systematic review of the literature. Dig Dis Sci 2013; 58:33893396. [CrossRef]
22. Jung ES, Kim JH, Yoon EL, et al. Comparison of the methods for tumor response assessment in patients with hepatocellular carcinoma undergoing transarterial chemoembolization. J Hepatol 2013; 58:1181-1187. [CrossRef]

23. Lencioni R. Loco-regional treatment of hepatocellular carcinoma. Hepatology 2010; 52:762773. [CrossRef]

24. Cheng AL, Amarapurkar D, Chao $\mathrm{Y}$, et al. Re-evaluating transarterial chemoembolization for the treatment of Hepatocellular Carcinoma: Consensus recommendations and review by an International Expert Panel. Liver Int 2014; 34:174-183. [CrossRef]

25. Niessen C, Wiggermann P, Velandia C, Stroszczynski C, Pereira PL. Transarterial chemoembolization - status quo in Germany. Rofo 2013; 185:1089-1094. [CrossRef]

26. Brown KT, Do RK, Gonen M, et al. Randomized trial of hepatic artery embolization for hepatocellular carcinoma using doxorubicin-eluting microspheres compared with embolization with microspheres alone. J Clin Oncol 2016; 34:2046-2053. [CrossRef]

27. Cammà CC, Schepis FF, Orlando AA, et al. Transarterial chemoembolization for unresectable hepatocellular carcinoma: meta-analysis of randomized controlled trials. Radiology 2002; 224:47-54. [CrossRef]

28. Wang P, Sheng L, Wang G, et al. Association of transarterial chemoembolization with survival in patients with unresectable hepatocellular carcinoma. Mol Clin Oncol 2014; 2:203-206.

29. Donati OF, Do RKG, Hötker AM, et al. Interreader and inter-test agreement in assessing treatment response following transarterial embolization for hepatocellular carcinoma. Eur Radiol 2015; 25:2779-2788. [CrossRef] 